\title{
The instability of periodic surface gravity waves
}

\author{
BERNARD DECONINCK ${ }^{1}$ AND KATIE OLIVERAS
}

${ }^{1}$ Department of Applied Mathematics, University of Washington, Seattle, WA 98195-2420, USA

${ }^{2}$ Mathematics Department, Seattle University, Seattle, WA 98122-1090, USA

(Received 11 March 2010; revised 30 September 2010; accepted 23 December 2010; first published online 17 March 2011)

Euler's equations describe the dynamics of gravity waves on the surface of an ideal fluid with arbitrary depth. In this paper, we discuss the stability of periodic travelling wave solutions to the full set of nonlinear equations via a non-local formulation of the water wave problem, modified from that of Ablowitz, Fokas \& Musslimani (J. Fluid Mech., vol. 562, 2006, p. 313), restricted to a one-dimensional surface. Transforming the non-local formulation to a travelling coordinate frame, we obtain a new formulation for the stationary solutions in the travelling reference frame as a single equation for the surface in physical coordinates. We demonstrate that this equation can be used to numerically determine non-trivial travelling wave solutions by exploiting the bifurcation structure of this new equation. Specifically, we use the continuous dependence of the amplitude of the solutions on their propagation speed. Finally, we numerically examine the spectral stability of the periodic travelling wave solutions by extending Fourier-Floquet analysis to apply to the associated linear non-local problem. In addition to presenting the full spectrum of this linear stability problem, we recover past well-known results such as the Benjamin-Feir instability for waves in deep water. In shallow water, we find different instabilities. These shallow water instabilities are critically related to the wavelength of the perturbation and are difficult to find numerically. To address this problem, we propose a strategy to estimate a priori the location in the complex plane of the eigenvalues associated with the instability.

Key words: instability, surface gravity waves

\section{Introduction}

Euler's equations for fluid motion are a set of nonlinear equations that describe surface gravity and capillary waves on an ideal fluid with arbitrary depth. A large class of commonly used mathematical models for ocean waves have been derived from these equations as approximations. Examples include the Korteweg-deVries equation (in the case of shallow water) and the nonlinear Schrödinger equation (in the case of deep water). While we can analytically determine solutions to some of the approximate equations, analytical solutions to the full set of Euler equations are unknown. Thus, we rely on alternative methods to determine information about the qualitative behaviour of solutions to the full equations such as bifurcation theory and stability analysis.

$\dagger$ Email address for correspondence: bernard@amath.washington.edu 
Already, there is a great wealth of literature discussing the stability of travelling water waves, and by no means can all aspects of it be covered completely within the context of this paper. One of the earliest results demonstrates that periodic waves in deep water are unstable with respect to long-wave perturbations. This modulational, or Benjamin-Feir, instability was discovered in the 1960s and reported on by Benjamin (1967), Benjamin \& Feir (1967) and Whitham (1967). By examining the Fourier mode expansion of the perturbed solution, Benjamin (1967) determined that small-amplitude waves with minimal period $2 \pi$ and dimensionless depth $k h>1.363$ are unstable with respect to long-wave perturbations.

More recently, numerical schemes have been used to determine the stability of travelling wave solutions with respect to various classes of perturbations. Some of the earliest results for the full water wave problem came in 1978. In two papers by Longuet-Higgins $(1978 a, b)$, the spectral stability of periodic travelling waves in infinite depth with respect to sub- and super-harmonic perturbations is investigated. This was achieved by calculating the first few Fourier modes of the solution to the perturbed equations, and numerically solving for the corresponding growth rates. Here, subharmonic and superharmonic refer to the period of the perturbation: harmonic perturbations share the period of the underlying wave, whereas the period of sub- (super-) harmonic perturbations is an integer multiple (quotient) of the period of the wave. In the early 1980s, the results of Longuet-Higgins $(1978 a, b)$ were extended to finite depth for transverse perturbations by McLean (1982a), and later by Kharif \& Ramamonjiarisoa (1990) and Francius \& Kharif (2006).

Even with many researchers working on the problem of stability of solutions to Euler's equations over the past 50 years, a large number of questions remain unanswered. First, to the best of our knowledge, there have been no publications or results demonstrating the full spectrum associated with the linearized problem (a recent paper by Nicholls 2009 presents a different method for determining the full spectrum of the linearized water wave problem. His method relies on the analytic dependence of the spectra on the amplitude of the solution.) Previous perturbation and numerical calculations were limited to a small number of Fourier modes or a small class of perturbations such as those presented in Benney \& Roskes (1969), McLean et al. (1981) and McLean (1982a,b). Additionally, for depths smaller than the critical threshold given by Benjamin (1967) and Whitham (1967), there has been little discussion of stability or instability with the exception of the recent work by Francius \& Kharif (2006).

In this paper, we aim to supplement past investigations on the instability of periodic travelling waves by using an extension of the non-local formulation of Ablowitz et al. (2006). Using this non-local formulation, we are able to reduce the equations of motion for a travelling wave to a single scalar equation in physical coordinates without approximation: in a travelling reference frame, the surface elevation of a gravity wave $\eta$ is determined by solving the equation

$$
\int_{0}^{L} \mathrm{e}^{-\mathrm{i} k x} \sqrt{\left(1+\eta_{x}^{2}\right)\left(c^{2}-2 g \eta\right)} \sinh (k(\eta+h)) \mathrm{d} x=0, \quad \forall k \in \Lambda,
$$

where $\Lambda$ is a lattice of appropriate wavenumbers, $L$ is the period of the solution and $h$ is the depth. A similar equation holds for capillary-gravity waves, see $\S 3$. With this single equation, we demonstrate a numerical scheme to determine the solution to the travelling wave equation to high degrees of accuracy.

Using this non-local formulation, we determine the stability of our solutions by using a Fourier-Floquet decomposition technique to find the full spectrum associated 


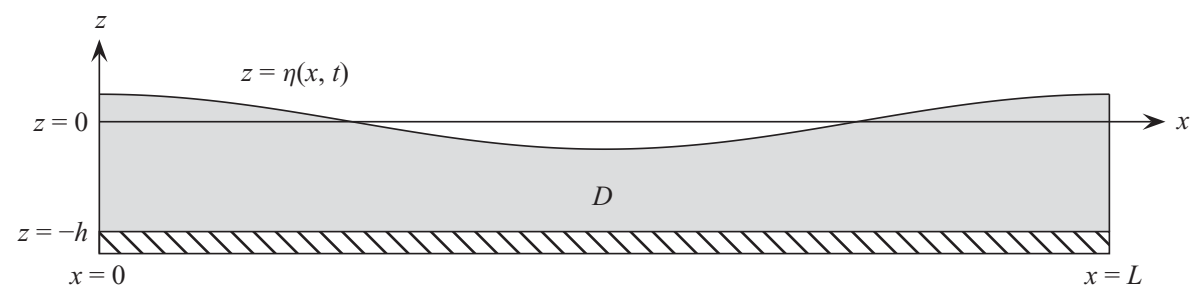

Figure 1. The fluid domain $D$.

with various travelling wave solutions. We find the expected result that for $k h>1.363$, waves are unstable to long-wave perturbations. In addition, we demonstrate the existence of instabilities in shallow water for waves of arbitrarily small amplitude with respect to one-dimensional perturbations travelling in the same direction as the underlying travelling wave.

Many considerations in this paper are valid for two-dimensional surfaces (or for surfaces with surface tension). However, contrary to the work of, for instance Benney \& Roskes (1969), McLean (1982a,b) and Francius \& Kharif (2006), we restrict ourselves to the stability of one-dimensional travelling waves with respect to one-dimensional perturbations. Our investigations of transverse perturbations and of capillary-gravity waves will be reported elsewhere. In this sense, our work can be considered a special case of theirs. We demonstrate that our results are in agreement with the limiting case of their one-dimensional perturbations. By specializing on one-dimensional perturbations without surface tension, we aim to provide a complete picture of this fundamentally important case. We are able to make new statements regarding the range and wavelength of the perturbations giving rise to shallow water instabilities, in addition to being able to compare the magnitude of the well-known Benjamin-Feir or modulational instability with that of other deep water instabilities.

\section{Equations of motion}

We consider Euler's equations for an ideal fluid with periodic boundary conditions restricted to a one-dimensional surface. For periodic boundary conditions, this requires us to solve Laplace's equation inside the fluid domain $D$ (see figure 1) with the appropriate boundary conditions. The governing equations for the fluid surface and velocity potential are given by

$$
\begin{gathered}
\phi_{x x}+\phi_{z z}=0, \quad(x, z) \in D, \\
\phi_{z}=0, \quad z=-h, \\
\eta_{t}+\eta_{x} \phi_{x}=\phi_{z}, \quad z=\eta(x, t), \\
\phi_{t}+\frac{1}{2}\left(\phi_{x}^{2}+\phi_{z}^{2}\right)+g \eta=\sigma \frac{\eta_{x x}}{\left(1+\eta_{x}^{2}\right)^{3 / 2}}, \quad z=\eta(x, t),
\end{gathered}
$$

where $\phi=\phi(x, z, t)$ is the velocity potential, $\eta=\eta(x, t)$ is the surface elevation, $g$ is the acceleration due to gravity, $h$ is the constant depth of the fluid when at a state of rest and $\sigma$ represents the coefficient of surface tension. We require periodic boundary conditions with period $L$. This provides additional boundary conditions on the surface elevation as well as the velocity potential once the induced mean flow has been eliminated, see Nicholls (1998). 
REMARK 1. We have assumed finite depth in the above formulation. The formulation is valid if $h \rightarrow \infty$ as well.

Euler's equations as written above are challenging to work with directly: they are a free-boundary problem with nonlinear boundary conditions. The complications include having to solve Laplace's equations inside an unknown domain, as well as having to deal with the highly nonlinear boundary conditions applied at the unknown free surface. To address these complications, several reformulations are useful. For one-dimensional surfaces, conformal mappings have been used to eliminate the problems mentioned, see for instance Okamoto \& Shoji (2001) and Dyachenko et al. (1996). Instead, we use an extension of the non-local formulation due to Ablowitz et al. (2006) which has two important advantages: (i) the equations of motion are posed in the original physical variables. Thus, a conformal mapping to and from an unknown domain is not required; (ii) the formulation is equally valid for three-dimensional fluids with two-dimensional surfaces. Other formulations (such as the Hamiltonian formulation due to Zakharov (1968) or the Zakharov-Craig-Sulem formulation of Craig \& Sulem 1993) eliminate some of the stated problems as well, by introducing a Dirichlet-to-Neumann operator (DNO) to reduce the Euler equations to a set of two partial differential equations in terms of surface variables. However, using their formulation one must truncate the series expansion of the DNO. The non-local formulation presented below does not require such a truncation.

Ablowitz et al. (2006) introduced a new, non-local formulation of the Euler equations, valid for surface waves localized on the whole line or the whole plane. It is almost trivial to extend this formulation to periodic boundary conditions. Restricting ourselves to a one-dimensional surface, the periodic generalization of the equations given by Ablowitz et al. (2006) is

$$
\begin{gathered}
q_{t}+\frac{1}{2} q_{x}^{2}+g \eta-\frac{1}{2} \frac{\left(\eta_{t}+\eta_{x} q_{x}\right)^{2}}{1+\eta_{x}^{2}}=\sigma \frac{\eta_{x x}}{\left(1+\eta_{x}^{2}\right)^{3 / 2}}, \\
\int_{0}^{L} \mathrm{e}^{-\mathrm{i} k x}\left(\mathrm{i} \eta_{t} \cosh (k(\eta+h))-q_{x} \sinh (k(\eta+h))\right) \mathrm{d} x=0, \quad \forall k \in \Lambda_{L},
\end{gathered}
$$

where $\Lambda_{L}$ is defined as the dual lattice to the period lattice of the wave, minus the origin:

$$
\Lambda_{L}=\left\{\frac{2 n \pi}{L} \mid n \in \mathbb{Z}-\{0\}\right\} .
$$

The periodic boundary conditions have been incorporated in the non-local equation by choosing $k \in \Lambda_{L}$. In (2.5) and (2.6), $q(x, t)$ is the velocity potential evaluated at the surface: $q(x, t)=\phi(x, \eta(x, t), t)$. The non-local equation (2.6), evaluated at all values of $k \in \Lambda_{L}$, gives an infinite number of algebraic conditions relating $\eta$ and $q$. Alternatively, and more conveniently, it can be thought of as a single scalar integrodifferential equation for $\eta$ and $q$, comparable to an equation obtained by equating all Fourier coefficients of a partial differential equation to zero.

For decaying boundary conditions as $|x| \rightarrow \infty$, the non-local formulation was shown to be equivalent to the original equations of motion by Ablowitz \& Haut (2008). Their proof extends to the periodic case in a straightforward way by comparing the periodic non-local formulation with the corresponding periodic formulation of the Zakharov-Craig-Sulem formulation of the water wave problem, see Craig \& Sulem (1993). The only modification necessary to the proof of Ablowitz \& Haut (2008) is 
that the equations of motion are formulated in a domain with prescribed harmonic functions at the side boundaries at the interior of the fluid.

REMARK 2. Equation (2.6) does not hold for $k=0$ : during its derivation, this equation is found multiplied by $k$. For convenience, this factor is removed, which can be done for all equations except the one with $k=0$. For $k=0$, the original equation states the triviality $0=0$, and presents no information. However, no harm is done by reintroducing (2.6) with $k=0$, as the corresponding equation simply presents the well-known statement of conservation of mass. It should be noted that this situation is different from the whole line case presented in Ablowitz et al. (2006). There, $k$ is a continuous parameter, and it is natural to consider the limit $k \rightarrow 0$ of (2.6), which naturally leads to a re-inclusion of $k=0$ as an admissible value. This limit process is not possible here, as the set of admissible $k$ values is discrete and $k=0$ is isolated.

\section{Travelling wave solutions}

Since we are interested in travelling wave solutions, we rewrite (2.5) and (2.6) in a coordinate system moving with speed $c$. Thus, we transform the independent variables $x$ and $t$ in the following way:

$$
\left(x^{\prime}, t\right) \rightarrow(x-c t, t)
$$

The system of equations we obtain by moving to the translating coordinates (omitting the primes) is

$$
\begin{gathered}
q_{t}-c q_{x}+\frac{1}{2} q_{x}^{2}+g \eta-\frac{1}{2} \frac{\left(\eta_{t}-c \eta_{x}+q_{x} \eta_{x}\right)^{2}}{1+\eta_{x}^{2}}=\sigma \frac{\eta_{x x}}{\left(1+\eta_{x}^{2}\right)^{3 / 2}} \\
\int_{c t}^{L+c t} \mathrm{e}^{-\mathrm{i} k(x-c t)}\left(\left(\eta_{t}-c \eta_{x}\right) \cosh (k(h+\eta))-\mathrm{i} q_{x} \sinh (k(h+\eta))\right) \mathrm{d} x=0, \quad \forall k \in \Lambda_{L} .
\end{gathered}
$$

Using the periodic boundary conditions, (3.3) simplifies to the following integration over the fundamental period:

$$
\int_{0}^{L} \mathrm{e}^{-\mathrm{i} k x}\left(\left(\eta_{t}-c \eta_{x}\right) \cosh (k(h+\eta))-\mathrm{i} q_{x} \sinh (k(h+\eta))\right) \mathrm{d} x=0, \quad \forall k \in \Lambda_{L} .
$$

To obtain stationary solutions, we equate the time derivative to zero. This gives the equations of motion for a stationary surface gravity wave in a moving reference frame

$$
\begin{gathered}
-c q_{x}+\frac{1}{2} q_{x}^{2}+g \eta-\frac{1}{2} \frac{\eta_{x}^{2}\left(q_{x}-c\right)^{2}}{1+\eta_{x}^{2}}=\sigma \frac{\eta_{x x}}{\left(1+\eta_{x}^{2}\right)^{3 / 2}}, \\
\int_{0}^{L} \mathrm{e}^{-\mathrm{i} k x}\left(-c \eta_{x} \cosh (k(h+\eta))-\mathrm{i} q_{x} \sinh (k(h+\eta))\right) \mathrm{d} x=0, \quad \forall k \in \Lambda_{L} .
\end{gathered}
$$

Solving these equations, we are interested mainly in determining the surface elevation $\eta(x, t)$. In the above formulation, we are required to solve two equations for the two unknown functions $\eta$ and $q$. This is further simplified by noting that (3.5) is a simple quadratic equation in $q_{x}$. This allows us to solve (3.5) for $q_{x}$, determining the $x$-derivative of the velocity potential at the surface explicitly in terms of the surface 
elevation $\eta$. We find

$$
q_{x}=c \pm \sqrt{\left(1+\eta_{x}^{2}\right)\left(c^{2}-2 g \eta+2 \sigma \frac{\eta_{x x}}{\left(1+\eta_{x}^{2}\right)^{3 / 2}}\right)}
$$

REMARK 3. When we transform the problem to a travelling wave coordinate frame, we induce a mean flow such that $q(x, t) \rightarrow q(x-c t, t)-c x$. One can change the problem to eliminate this unbounded growth of the velocity potential in the travelling frame by introducing a new velocity potential $\tilde{q}$ at the surface. In the original nontravelling coordinates $\tilde{q}(x-c t, t)=q(x-c t, t)-c x$. Then, $\tilde{q}$ is bounded for all $(x, t)$. This new velocity potential $\tilde{q}$ satisfies

$$
\tilde{q}_{x}= \pm \sqrt{\left(1+\eta_{x}^{2}\right)\left(c^{2}-2 g \eta+2 \sigma \frac{\eta_{x x}}{\left(1+\eta_{x}^{2}\right)^{3 / 2}}\right)}
$$

Introducing $\tilde{q}_{x}$, the meaning of the plus/minus sign is clear. It refers to right-and lefttravelling waves. For simplicity, we choose the plus sign. We note there are no differences between the two cases.

With this expression for the velocity potential at the surface, we simplify (3.6). Substituting (3.7) in (3.6) followed by integrating by parts, we obtain a single scalar non-local equation for the surface elevation $\eta$

$$
\int_{0}^{L} \mathrm{e}^{-\mathrm{i} k x} \sqrt{\left(1+\eta_{x}^{2}\right)\left(c^{2}-2 g \eta+2 \sigma \frac{\eta_{x x}}{\left(1+\eta_{x}^{2}\right)^{3 / 2}}\right)} \sinh (k(\eta+h)) \mathrm{d} x=0, \quad \forall k \in \Lambda_{L} .
$$

Similarly, for the case of infinite depth, these same steps are repeated to obtain

$$
\int_{0}^{L} \mathrm{e}^{-\mathrm{i} k x} \sqrt{\left(1+\eta_{x}^{2}\right)\left(c^{2}-2 g \eta+2 \sigma \frac{\eta_{x x}}{\left(1+\eta_{x}^{2}\right)^{3 / 2}}\right)} \mathrm{e}^{|k| \eta} \mathrm{d} x=0, \quad \forall k \in \Lambda_{L} .
$$

Thus, we are presented with a single scalar non-local equation determining the stationary periodic water wave profiles in either water of depth $h$, or water of infinite depth. This equation provides a compact formulation for the stationary water wave problem, especially if surface tension is neglected $(\sigma=0)$. It is written down using the original physical variables, and it only requires solving for the main unknown of interest, $\eta$. Once a solution $\eta$ is determined, the velocity potential at the surface is obtained directly by substitution in (3.7).

REMARK 4. The use of periodic boundary conditions was not essential to obtain equations (3.9) and (3.10). These equations, with $k \in \mathbb{R}-\{0\}$ and $x \in \mathbb{R}$ are equally valid on the whole line. The equations appear to be new for that case as well.

Using the above formulation, we can draw some immediate conclusions about the periodic travelling waves of the Euler equations. For example, let $\eta_{\max }=a$ represent the maximum height above the average value of a gravity wave with zero surface tension $(\sigma=0)$. We know that $\eta_{x}=0$ at the crest for smooth waves. It follows that, 
in order for the velocity potential to remain real valued

$$
a \leqslant \frac{c^{2}}{2 g},
$$

which is the same inequality given by Craig \& Sternberg (1988). It is obtained here using more elementary methods. For the remainder of this paper, we consider the case of pure gravity waves and equate $\sigma=0$.

\subsection{Constructing solutions: numerical implementation}

We numerically determine the periodic travelling wave solutions to the fully nonlinear equations, see for example Cokelet (1977), Rienecker \& Fenton (1981), VandenBroeck (1983), Tanaka (1983), Chandler \& Graham (1993) and Craig \& Nicholls (2002). Specifically, we investigate how the speed, $c$, of the nontrivial solutions varies for different wave amplitudes. This type of bifurcation problem is set up ideally for a numerical continuation and has been explored using the DNO formulation by Nicholls (1998) and Craig \& Nicholls (2002). We choose a similar strategy for determining the numerical solutions of the non-local equations (3.9) and (3.10), with $\sigma=0$.

To implement our numerical continuation method, we use a pseudospectral method where differentiation is computed in Fourier space, and nonlinear operations are computed in physical space. Since we are considering periodic solutions, it is natural to choose a projection of the solution $\eta$ onto a truncated Fourier series representation with $N$ Fourier modes. This gives an approximation for the surface elevation $\eta$ at our collocation points $x_{j}$ as

$$
\eta\left(x_{j}\right) \approx \sum_{n=-N}^{N} \hat{\eta}_{n} \mathrm{e}^{\mathrm{i} k_{n} x_{j}},
$$

in terms of the $2 N+1$ unknown Fourier coefficients $\hat{\eta}_{n}$. We can reduce the number of unknown Fourier coefficients by noting that the average value of the surface elevation can be fixed without loss of generality by renormalizing the value of the depth $h$. This allows us the freedom to choose the zeroth Fourier coefficient of the surface elevation. For simplicity, we let $\hat{\eta}_{0}=0$. This leaves us with $2 N$ unknown Fourier coefficients.

The non-local spectral equation (3.9) (and (3.10) for infinite depth) is valid for all $k \in \Lambda_{L}$, with $\Lambda_{L}=\{2 n \pi / L \mid n \in \mathbb{Z}-\{0\}\}$. Again, we have chosen to ignore the equation for $k=0$ since it is identically satisfied. The physical significance of that equation (conservation of mass) has been incorporated by choosing the zeroth Fourier coefficient of the surface elevation to be identically zero. Since we have chosen to truncate our Fourier expansion of $\eta$ yielding $2 N$ unknown coefficients, we incorporate the $2 N$ equations corresponding to $n=-N, \ldots,-1,1, \ldots, N$ yielding $2 N$ equations for $2 N$ unknowns.

In order to solve for the travelling wave solution with various amplitudes, we consider the bifurcation problem where the amplitude parameter $a$ is varied, and the velocity parameter $c$ is regarded as a function of $a$. This introduces the new unknown $c$, requiring the introduction of a new equation, parameterized by a fixed value of the surface elevation $a=\eta_{\max }$. This extra equation is simply the statement that the infinity norm of the solution is equal to $a$. Thus, now we are charged with solving the 

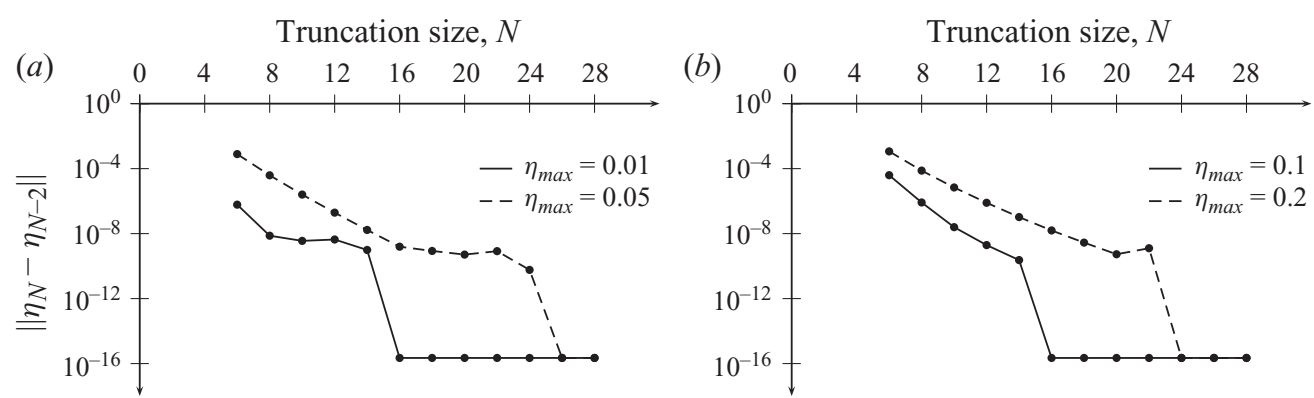

FIgURE 2. Semilog plot of the Cauchy error for various depths $(h=0.5(a)$ and $h=\infty(b))$ for two different amplitudes, illustrating a spectral convergence rate.

$2 N+1$ equations given by

$$
\begin{gathered}
\int_{0}^{L} \mathrm{e}^{-\mathrm{i} k x} \sqrt{\left(1+\eta_{x}^{2}\right)\left(c^{2}-2 g \eta\right)} \sinh (k(\eta+h)) \mathrm{d} x=0, \quad k \in \Lambda_{L}^{(N)}, \\
\|\eta\|_{\infty}=a,
\end{gathered}
$$

for the unknowns $c$ and $\hat{\eta}_{n}$, where $\Lambda_{L}^{(N)}=\{2 n \pi / L \mid n \in-N, \ldots,-1,1, \ldots, N\}$.

Equations (3.13) and (3.14) are solved for the surface elevation iteratively via Newton's method (though other iterative techniques can also be used). Using numerical continuation, we determine travelling wave solutions for increasingly larger amplitudes until the critical wave height is reached as determined numerically by the high-precision calculations of Cokelet (1977).

One of the main advantages of our formulation is that we need to numerically solve only for the surface elevation $\eta$ and wave speed $c$. Once we know the surface elevation, we can explicitly determine the velocity potential at the surface from (3.7). Other commonly used numerical methods either require the simultaneous solution of the velocity potential or a secondary iterative procedure to solve for the velocity potential, see Longuet-Higgins (1988) and Francius \& Kharif (2006). By eliminating the velocity potential, we are able to greatly reduce the complexity of our numerical computations.

\subsection{Constructing solutions: numerical results}

Before we demonstrate the numerical results obtained using the continuation method, we discuss convergence and stability of the algorithm. We do not attempt to prove convergence of the scheme analytically. Instead, we demonstrate that as the number of Fourier modes used to approximate the surface elevation is increased, the Fourier coefficients of each successive approximation converge to the same solution. Let $\eta_{N}$ represent the solution $\eta$ computed with $N$ Fourier modes (i.e. $2 N+1$ collocation points). Define the error $e_{N}$ as

$$
e_{N}=\left\|\eta_{N}-\eta_{N-2}\right\|_{2}
$$

We demonstrate Cauchy convergence by showing that the difference in the approximations of the solution converges to $\epsilon_{t o l}$ where $\epsilon_{t o l}$ is the machine-determined round-off error. For several amplitudes and depths, we display the Cauchy error as a function of the number of Fourier modes used in the approximation of the solution, as shown in figure 2. Clearly, the Cauchy error decreases linearly on a logarithmic scale which demonstrates spectral accuracy. It is important to note that as the amplitude of the solution increases (and consequently the nonlinearity of the 


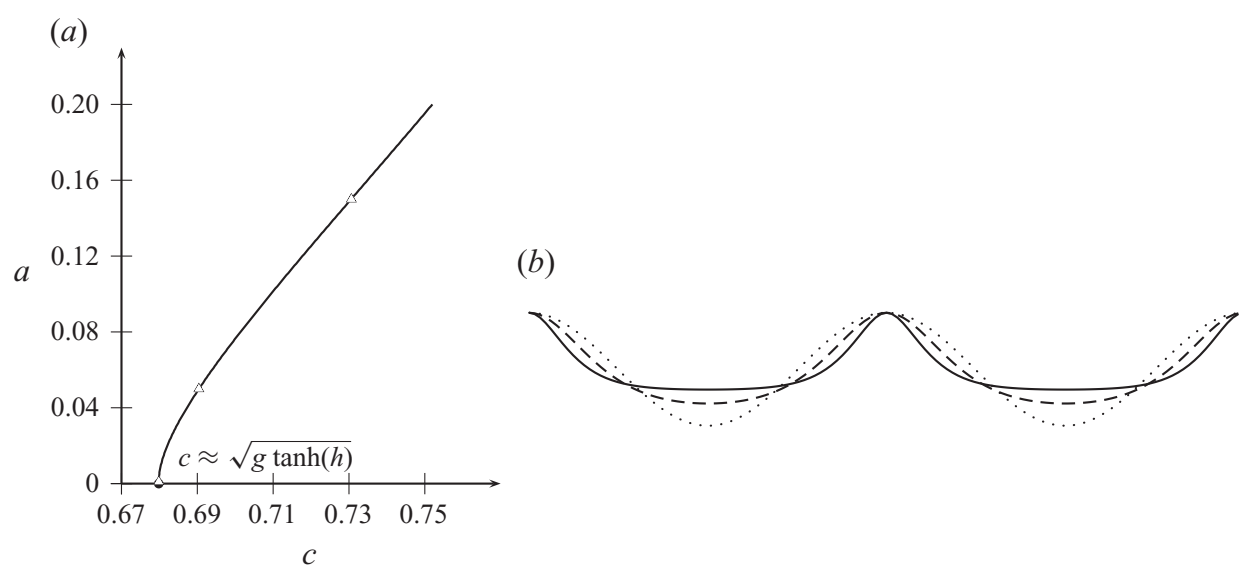

FiguRE 3. (a) The bifurcation curve of amplitude versus speed of the travelling wave solution for $h=0.5$. ( $b$ ) Plots of the rescaled travelling wave solution $\eta / a$ for amplitudes $a=0.001$ (dotted line), $a=0.05$ (dashed line) and $a=0.15$ (solid line). The corresponding points for the travelling wave solutions are indicated on the bifurcation curve. All solutions are calculated with $N=64$ Fourier modes with residual error less than $10^{-14}$ and plotted over two periods for clarity.

solution), it is necessary to take a larger number of Fourier modes to attain an equally small Cauchy error.

Now that we have illustrated the Cauchy convergence of our numerical scheme, we proceed to calculate travelling wave solutions for a variety of parameters as needed for the stability analysis in the next section. Our numerical solutions demonstrate the phenomena we expect. For example, in shallow water, as we increase the amplitude, the solutions become more elliptic. In other words, for increasing amplitude, the troughs widen and the crests become sharper as demonstrated in figure 3 . The amplitude of the wave profiles shown in figure 3 is rescaled to 1 , to allow for easier comparison of the profiles.

For waves in deep water, the results are illustrated in figures 4 and 5. As before, the amplitude of the wave profiles has been rescaled to 1 for easier comparison. In figure $4, h=1.5$, while in figure 5 we have $h=\infty$. As expected, although the wave becomes steeper and profiles develop sharper crests as the nonlinearity increases, no extensive troughs develop as happens in shallow water. The bifurcation curve for $h=1.5$ barely shows the pronounced (but smooth) corner visible for $h=\infty$. Notice the similarity between the wave profiles in deep water of depth $h=1.5$ and depth $h=\infty$.

We are able to calculate solutions to $99 \%$ of the maximal wave height corresponding to the $120^{\circ}$ crest. However, since we are using Fourier series to determine the solutions numerically, it is expected that our method breaks down near the limiting wave height where the solutions are no longer smooth, and the method loses its spectral convergence rate. This is a limitation of our current numerical method. Regardless, we are still able to examine almost the entire parameter space very efficiently using our current implementation. A discussion on the convergence of the Fourier series of the steepest wave is given by Plotnikov \& Toland (2002).

\section{Stability formulation}

We investigate the spectral stability of the travelling wave profiles computed in the previous section with respect to infinitesimal perturbations. First, it is important that 


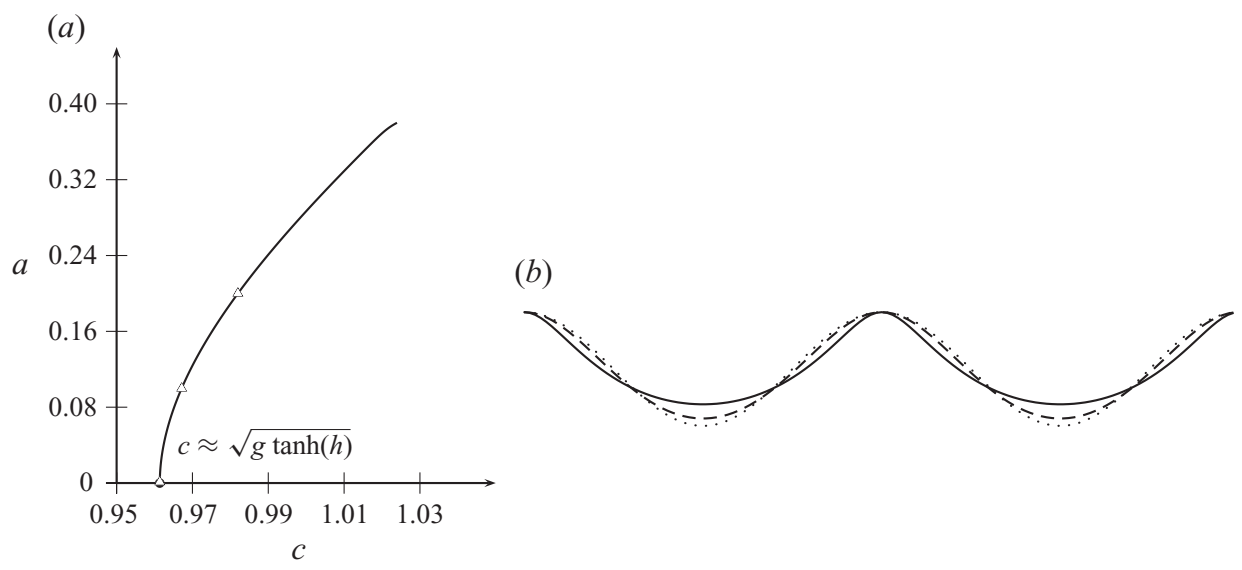

FIGURE 4. The bifurcation curve of amplitude versus speed of the travelling wave solution for $h=1.5(a)$. Plots of the rescaled travelling wave solution $\eta / a(b)$ for amplitudes $a=0.001$ (dotted line), $a=0.02$ (dashed line) and $a=0.2$ (solid line). The corresponding points for the travelling wave solutions are indicated on the bifurcation curve. All solutions are calculated with $N=64$ Fourier modes with residual error less than $10^{-14}$ and plotted over two periods for clarity.

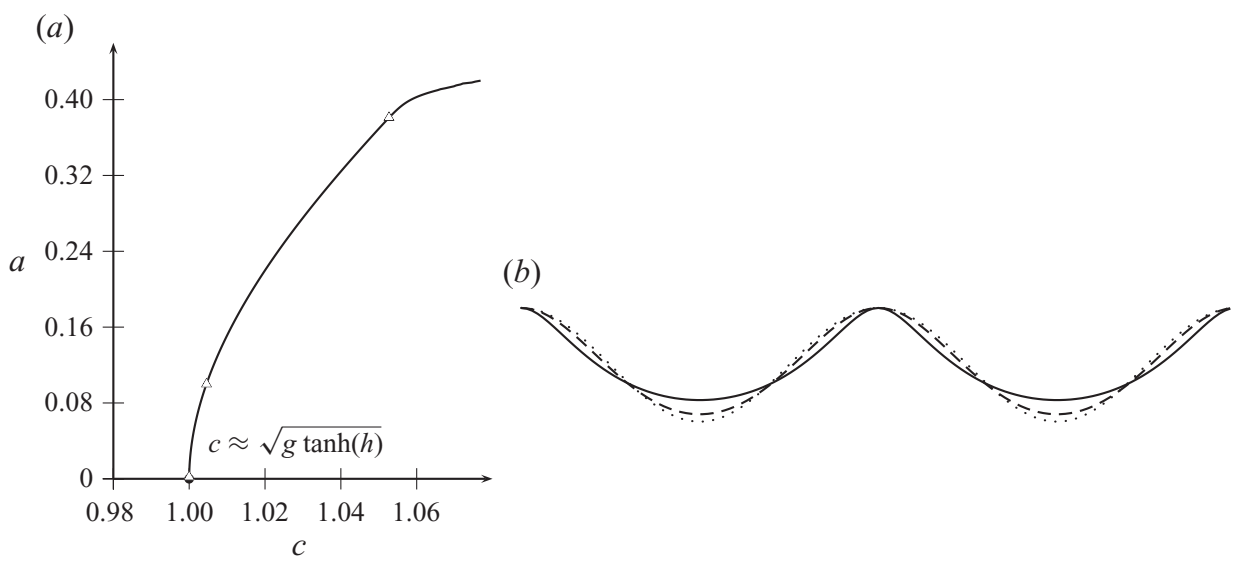

FIGURE 5. The bifurcation curve of amplitude versus speed of the travelling wave solution for $h=\infty(a)$. Plots of the rescaled travelling wave solution $\eta / a(b)$ for amplitudes $a=0.001$ (dotted line), $a=0.01$ (dashed line) and $a=0.381$ (solid line). The corresponding points for the travelling wave solutions are indicated on the bifurcation curve. All solutions are calculated with $N=64$ Fourier modes with residual error less than $10^{-14}$ and plotted over two periods for clarity.

we discuss what perturbations we wish to allow. It may appear natural to consider disturbances of the same period as the underlying stationary wave, as is often done in the literature. However, we wish to work with a more general class of disturbances; namely, we choose to include all bounded on the whole real line. Thus, for us ||$U(x)||<\infty$ means $\sup _{\mathbb{R}}|U(x)|<\infty$ and $U(x)$ continuous for all $x \in \mathbb{R}$. In other words, $U(x) \in C_{b}^{0}(\mathbb{R})$. It is important to realize that this class is the largest class of perturbations allowed by the physical problem at hand. Indeed, disturbances should be bounded and continuous functions, but there is no physical reason to restrict their spatial dependence to be periodic. 


\subsection{Stability formulation: problem reformulation}

In order to investigate the stability of the travelling wave profiles with respect to such perturbations, it is necessary to reformulate the governing equations. Equation (2.5) is a local statement and does not require modification. However, (2.6) is non-local and in its current incarnation applies specifically to waves of period $L$. Thus, we cannot use the equation in its current form for any bounded function on the real line.

To address this problem, it is necessary to reformulate the non-local equation. Let $\langle f\rangle$ represent the spatial average of a function $f(x), x \in \mathbb{R}$ defined by

$$
\langle f\rangle=\lim _{M \rightarrow \infty} \frac{1}{M} \int_{-M / 2}^{M / 2} f(x) \mathrm{d} x .
$$

It is clear that if $f(x)$ is periodic with period $L$, then $\langle f\rangle$ is well defined. But the spatial average is well defined for functions that are not periodic as well, such as the quasi-periodic functions, see Bohr (1947). In fact, the spatial average is defined for all functions in $C_{b}^{0}(\mathbb{R})$. It should be noted that the kernel of this operation is quite large. For instance, all functions that approach zero as $|x| \rightarrow \infty$ have zero spatial average. Nevertheless, we may use the spatial average to replace (2.6) with the more general non-local equation

$$
\left\langle\mathrm{e}^{-\mathrm{i} k x}\left(\mathrm{i} \eta_{t} \cosh (k(\eta+h))+q_{x} \sinh (k(\eta+h))\right)\right\rangle=0,
$$

valid for all $k \in \mathbb{R}_{0}=\mathbb{R}-\{0\}$, as no quantization condition is imposed by the periodicity of the solutions considered. In fact, as solutions of increasingly larger period are considered, the set of $k$ values to be considered in (2.6) approaches a dense subset of the real line. The equation corresponding to $k=0$ is excluded, as before. Equation (4.2) allows us to perturb our travelling wave solution with any bounded perturbation regardless of periodicity.

\subsection{Stability formulation: eigenvalue problem}

Having generalized the dynamical equations to accommodate the perturbations we wish to consider, we briefly discuss the definition of spectral stability. A stationary solution of a nonlinear problem is spectrally stable if there are no exponentially growing modes of the corresponding linearized problem. To determine the spectral stability of the periodic travelling wave solutions, we start by considering a travelling wave solution set $\left(\eta_{0}(x-c t), q_{0}(x-c t)\right)$ which solves (3.9) (or (3.10) in the case of infinite depth). In the same travelling coordinate frame, we add a small perturbation of the form

$$
\begin{aligned}
& q(x-c t, t)=q_{0}(x-c t, t)+\epsilon q_{1}(x-c t) \mathrm{e}^{\lambda t}+O\left(\epsilon^{2}\right), \\
& \eta(x-c t, t)=\eta_{0}(x-c t, t)+\epsilon \eta_{1}(x-c t) \mathrm{e}^{\lambda t}+O\left(\epsilon^{2}\right),
\end{aligned}
$$

where $\epsilon$ is a small parameter. The perturbations $\eta_{1}$ and $q_{1}$ are moving at the same speed and in the same direction as the original travelling wave solution. Our goal is to determine the time dependence of the perturbation in order to determine how the deviation from the unperturbed solution evolves.

For simplicity, we replace $x-c t$ with $x$, where $\eta$ and $q$ are periodic in the new variable $x$ with period $2 \pi$. Since we are linearizing about a solution, the terms at 
$O\left(\epsilon^{0}\right)$ vanish, and the leading-order terms of the equation at $O(\epsilon)$ are

$$
\begin{gathered}
\lambda\left(q_{1}-f_{1}\left(\eta_{0}, q_{0}\right) \eta_{1}\right)=-\left(q_{0, x}-c\right) q_{1, x}-g \eta_{1}-f_{2}\left(\eta_{0}, q_{0}\right) \eta_{1, x} \\
+f_{1}\left(\eta_{0}, q_{0}\right)\left(\left(q_{0, x}-c\right) \eta_{1, x}+\eta_{0, x} q_{1, x}\right), \\
\mathrm{i} \lambda\left\langle\mathrm{e}^{-\mathrm{i} k x} \eta_{1} \mathscr{C}_{k}\right\rangle=\left\langle\mathrm{e}^{-\mathrm{i} k x}\left(k\left(q_{0, x}-c\right) \eta_{1, x} \mathscr{C}_{k}+q_{1, x} \mathscr{I}_{k}\right)\right\rangle,
\end{gathered}
$$

for all $k \in \mathbb{R}_{0}$, where for brevity

$$
\begin{gathered}
f_{1}\left(\eta_{0}, q_{0}\right)=\frac{\eta_{0, x}\left(q_{0, x}-c\right)}{1+\eta_{0, x}^{2}}, \quad f_{2}\left(\eta_{0}, q_{0}\right)=f_{1}^{2}\left(\eta_{0}, q_{0}\right), \\
\mathscr{S}_{k}=\sinh \left(k\left(\eta_{0}+h\right)\right), \quad \text { and } \quad \mathscr{C}_{k}=\cosh (k(\eta+h)) .
\end{gathered}
$$

This is rewritten compactly as

$$
\lambda \mathscr{L}_{1} U(x)=\mathscr{L}_{2} U(x)
$$

where $\mathscr{L}_{1}$ and $\mathscr{L}_{2}$ are $2 \times 2$ matrices of linear operators, whose entries are easily read off from (4.5) and (4.6).

Since the time dependence of the perturbation depends exponentially on $\lambda$, we can determine information about the stability of the underlying travelling wave by determining all bounded solutions of this generalized eigenvalue problem. If any bounded solutions $U(x)$ exist for which the corresponding $\lambda$ has a positive real part, the linear approximation of the solution will grow in time and thus the perturbed solution will exponentially diverge from the stationary solution in the linear approximation.

Since the coefficient functions of (4.9) are periodic in $x$ with period $L$, we decompose the perturbations further using Floquet's Theorem, see for instance Deconinck \& Kutz (2006). This allows us to write

$$
q_{1}(x)=\mathrm{e}^{\mathrm{i} \mu x} \bar{q}_{1}(x), \quad \text { and } \quad \eta_{1}(x)=\mathrm{e}^{\mathrm{i} \mu x} \bar{\eta}_{1}(x),
$$

where $\bar{q}_{1}(x)$ and $\bar{\eta}_{1}(x)$ are periodic with period $2 \pi$ and $\mu$ can be restricted to the interval $[-1 / 2,1 / 2]$.

Substituting the Floquet decomposition directly into (4.5), we obtain

$$
\begin{gathered}
\lambda\left(\bar{q}_{1}-f_{1}\left(\eta_{0}, q_{0}\right) \bar{\eta}_{1}\right)=\left(c-q_{0, x}\right)\left(\mathrm{i} \mu+\partial_{x}\right) \bar{q}_{1}-g \bar{\eta}_{1}-f_{2}\left(\eta_{0}, q_{0}\right)\left(\mathrm{i} \mu+\partial_{x}\right) \bar{\eta}_{1} \\
+f_{1}\left(\eta_{0}, q_{0}\right)\left(\left(q_{0, x}-c\right)\left(\mathrm{i} \mu+\partial_{x}\right) \bar{\eta}_{1}+\eta_{0, x}\left(\mathrm{i} \mu+\partial_{x}\right) \bar{q}_{1}\right) .
\end{gathered}
$$

As expected for a differential operator, the Floquet multiplier $\exp (\mathrm{i} \mu x)$ factors from the equation, leaving only remnant Floquet exponents $\mu$, depending on the differential order of a specific term. For the non-local equation (4.6), this does not happen, and extra care is required. Substituting the Floquet decomposition into the non-local equation (4.6), we find

$$
\lambda\left\langle\mathrm{e}^{-\mathrm{i}(k-\mu) x} \mathscr{C}_{k} \bar{\eta}_{1}\right\rangle=\left\langle\mathrm{e}^{-\mathrm{i}(k-\mu) x}\left(k\left(\mathrm{i} \mu+\partial_{x}\right)\left(q_{0, x}-c\right) \bar{\eta}_{1} \mathscr{C}_{k}+\left(\mathrm{i} \mu+\partial_{x}\right) \bar{q}_{1} \mathscr{S}_{k}\right)\right\rangle .
$$

We represent the $2 \pi$-periodic parts $\bar{q}_{1}$ and $\bar{\eta}_{1}$ of the eigenfunctions by their Fourier series. In other words

$$
\bar{q}_{1}(x)=\sum_{m=-\infty}^{\infty} \hat{Q}_{m} \mathrm{e}^{\mathrm{i} m x}, \quad \text { and } \quad \bar{\eta}_{1}(x)=\sum_{m=-\infty}^{\infty} \hat{N}_{m} \mathrm{e}^{\mathrm{i} m x} .
$$

The different coefficient functions are represented in a similar way. Substituting these expressions into (4.11) and (4.12), we determine a bi-infinite matrix-generalized eigenvalue problem, where the components of the eigenvectors are given by the Fourier coefficients $\hat{Q}_{j}$ and $\hat{N}_{j}$ for $j \in \mathbb{Z}$. Obtaining the Fourier decomposition for 
the local problem (4.5) is straightforward and completely analogous to the examples treated in Deconinck \& Kutz (2006). However, as announced, the decomposition for the non-local equation requires more care.

Substituting the Fourier series into (4.12), we have

$$
\begin{gathered}
{\left[\sum_{j=-\infty}^{\infty} \sum_{m=-\infty}^{\infty} \hat{G}_{1, j}^{(k, m, \mu)} \hat{N}_{m}+\sum_{j=-\infty}^{\infty} \sum_{m=-\infty}^{\infty} \hat{G}_{2, j}^{(k, m, \mu)} \hat{Q}_{m}\right]\langle E\rangle} \\
\quad=\mathrm{i} \lambda \sum_{j=-\infty}^{\infty} \sum_{m=-\infty}^{\infty} \hat{G}_{3, j}^{(k, m, \mu)} \hat{N}_{m}\langle E\rangle,
\end{gathered}
$$

where $\hat{G}_{j, n}^{(k, m, \mu)}$ represent the Fourier expansions of the appropriate coefficient function, and $E$ is given by $E=\exp (-\mathrm{i}(k-\mu) x+m x+j x)$. Each term in the doubly-infinite sum contains the average value of $E$. It is important to note $\langle E\rangle$ is identically zero unless the exponent is a multiple of $2 \pi \mathrm{i}$. In other words, the contribution from the integral vanishes unless

$$
k=\mu+n, \quad n \in \mathbb{Z}
$$

Thus, the only contribution to the integral equation occurs when the $k$-lattice is a shift of the original dual lattice. Substituting this relationship into the exponent, the remaining integral is

$$
\frac{1}{M} \int_{-M / 2}^{M / 2} \exp [\mathrm{ix}(j+m-n)] \mathrm{d} x
$$

which is identically zero unless $j+m-n=0$ and 1 otherwise. This allows us to collapse the doubly-infinite sum to a single bi-infinite sum given by

$$
\begin{aligned}
& \sum_{m=-\infty}^{\infty} \hat{G}_{1, n-m}^{(k(\mu), m)} \hat{N}_{m}+\sum_{m=-\infty}^{\infty} \hat{G}^{(k(\mu), m)} \hat{G}_{2, n-m}^{(k(\mu), m)} \hat{Q}_{m} \\
& =\mathrm{i} \lambda \sum_{m=-\infty}^{\infty} \hat{G}_{3, n-m}^{(k(\mu), m)} \hat{N}_{m},
\end{aligned}
$$

which holds for all $n \in \mathbb{Z}$.

Combining the expansion for the local equation with (4.17) gives a generalized bi-infinite eigenvalue problem for determining the spectrum of the linearized operator about the stationary travelling wave solutions.

We solve this generalized eigenvalue problem numerically by truncating the Fourier series representation from $\mathbb{Z}$ to $\{0, \pm 1, \pm 2, \ldots, \pm N\}$. With this truncation, we obtain $2(2 N+1)$ equations for $2(2 N+1)$ unknown Fourier coefficients $\hat{Q}_{n}$ and $\hat{N}_{n}$. Finally, we note that due to the underlying symmetries in the problem, we may restrict the $\mu$ interval from $[-1 / 2,1 / 2]$ to $[0,1 / 2]$.

\subsection{Stability formulation: convergence results}

To demonstrate the reliability of this numerical method, we show that the approximations of a particular eigenvalue converge as we increase the size of the truncated problem. The convergence and reliability of this truncation were investigated by Curtis \& Deconinck (2010) in general. Those results do no formally apply to our setting, but the results are easily generalized once we note that the appropriate matrix is invertible. (Since we are solving a generalized eigenvalue problem of the form $\boldsymbol{M} \boldsymbol{X}=\lambda \boldsymbol{N} \boldsymbol{X}$, we must worry about the invertibility of the matrix $\boldsymbol{N}$. For the AFM 
$N$

3

3
4

5

6

7

8

$$
h=0.5
$$

$5.1036 \times 10^{-5}$

$3.7838 \times 10^{-7}$

$4.6025 \times 10^{-11}$

$3.6857 \times 10^{-13}$

$1.8532 \times 10^{-15}$

$3.1619 \times 10^{-16}$

$h=1.5$
$7.3966 \times 10^{-6}$
$7.9354 \times 10^{-12}$
$2.4010 \times 10^{-15}$
$1.7606 \times 10^{-16}$
$1.2409 \times 10^{-15}$
$6.0266 \times 10^{-16}$

$h=1.5$

$2.9912 \times 10^{-6}$
$7.6283 \times 10^{-10}$
$2.1943 \times 10^{-15}$
$1.4463 \times 10^{-16}$
$1.2286 \times 10^{-16}$
$7.2879 \times 10^{-16}$

TABLE 1. Cauchy error for the calculation of the eigenvalue with largest real part for $h=0.5$, and for the calculation of two eigenvalues for $h=1.5$.

(a)

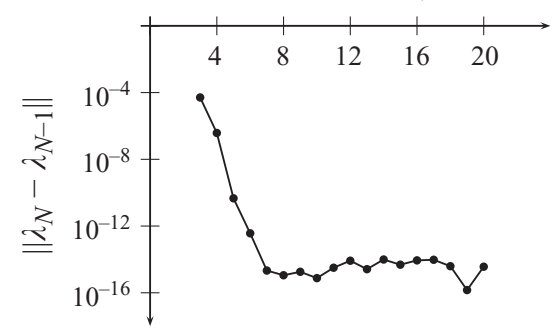

(b)

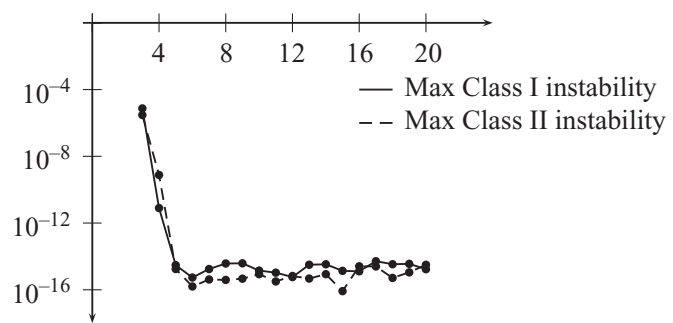

FIGURE 6. Semilog plot of the Cauchy error for various depths $(h=0.5(a)$ and $h=1.5(b))$ for $a=0.01$ illustrating a spectral convergence rate. For $h=1.5$, the convergence rates were calculated for the dominant Class I and Class II instabilities with $m=1$ (see below).

formulation, the matrix $\boldsymbol{N}$ is in block form and it follows that the singularity of $\boldsymbol{N}$ is determined by one block matrix which we numerically determine to have a non-zero determinant). Alternatively, we are able to demonstrate Cauchy convergence for the numerical method, demonstrating that we can trust the numerical results we obtain. For several parameters, we tracked the approximation of the eigenvalues with the largest real part as a function of truncation size. The Cauchy errors are given in table 1.

As is clearly seen in figure 6, the Cauchy error decreases linearly on a logarithmic scale which indicates spectral accuracy. Additionally, the figure shows that it is not necessary to consider large truncation values. Thus, in general, we start with a small number of Fourier modes. We note that as the nonlinearity of the travelling wave solution increases (the amplitude $a$ ), a larger number of Fourier modes is necessary for convergence. In the case of multiple eigenvalues with maximal real part, Cauchy convergence for the dominant growth rates can still be demonstrated as seen in figure 6 .

\section{Spectral instability results}

In this section, we present the numerical results of our stability investigations, and we discuss the characteristics of the instabilities found. We begin by demonstrating the full stability spectrum for several parameter choices in water of depth $h$ and amplitude $a$. We specifically choose to investigate three depths: $h=0.5, h=1.5$ and $h=\infty$. These choices give us two depths above and one depth below the critical Benjamin-Feir threshold discussed in the Introduction. Recall that throughout, we equate the period of the periodic travelling wave to $2 \pi$. 


\subsection{Adaptive selection of the values of $\mu$}

Before we proceed to the general results, we comment on the choice of $\mu$ values for which we calculate the spectrum. From the above, the spectrum should be computed for all values of $\mu$ in the interval $[-1 / 2,1 / 2]$. Previously, researchers worked with a coarse set of approximately 100-500 equally spaced values in this interval, see for instance Nicholls (2009). However, we have found that important information (including the distinction between stable and unstable waves) can be lost by choosing such a coarse grid. There are several methods to address this problem. One option is to choose a uniform but sufficiently fine mesh. The disadvantage of this approach is that it is not a priori clear what constitutes sufficiently fine, but mainly that this results in a number of computations that far exceeds the number necessary to capture the important information. We work with the second option of constructing a nonuniform set of $\mu$ values, adaptively chosen to capture the information important for stability considerations, as suggested by others (McLean 1982a; Francius \& Kharif 2006) and recapitulated below.

We start by considering the spectrum for the trivial solution where $\eta=0, q=0$ and $c=\sqrt{g \tanh h}$. In other words, we are considering the trivial solution at the base of the bifurcation branch. Since the linear stability problem for this solution has constant coefficients, we easily determine the spectrum analytically. We consider perturbations of the form

$$
\begin{aligned}
& \eta(x)=0+\epsilon \mathrm{e}^{\mathrm{i} \mu x+\lambda t} \sum_{j=-\infty}^{\infty} \hat{\eta}_{j} \mathrm{e}^{\mathrm{i} j x}, \\
& q(x)=0+\epsilon \mathrm{e}^{\mathrm{i} \mu x+\lambda t} \sum_{j=-\infty}^{\infty} \hat{q}_{j} \mathrm{e}^{\mathrm{i} j x},
\end{aligned}
$$

where $\epsilon$ is a small parameter. This perturbation form is the same form justified in the previous section, and hence we are able to capture the full spectrum. As expected, the eigenvalues $\lambda$ are purely imaginary. They are given by

$$
\lambda_{m}^{s}=-\mathrm{i}(c(\mu+m)+s \sqrt{(\mu+m) \tanh (\mu+m)}),
$$

where $s= \pm 1, m \in \mathbb{Z}$ and $\mu+m \neq 0$.

We wish to determine how the spectrum changes as we increase the amplitude of the solution. Using the results of MacKay \& Saffman (1986), we know that for an instability to arise for our specific Hamiltonian system, it is necessary for two eigenvalues with opposite signatures to collide. Since the signature of a particular eigenvalue is given by $\operatorname{sign}\left(\lambda_{m}^{s}\right)=-\operatorname{Im}\left(s \lambda_{m}^{s}\right)$, we can determine the possible location of eigenvalues giving rise to an instability by looking for two colliding eigenvalues $\lambda_{m}^{s_{1}}$ and $\lambda_{n}^{s_{2}}$ with opposite signatures

$$
\lambda_{m}^{s_{1}}=\lambda_{n}^{s_{2}} \quad \text { and } \quad-\operatorname{Im}\left(s_{1} \lambda_{m}^{s_{1}}\right)=\operatorname{Im}\left(s_{2} \lambda_{n}^{s_{2}}\right)
$$

If we solve these two equations, we immediately see that $s_{1}=-s_{2}$. Furthermore, one checks that collisions of opposing signatures occur when $n=-m$ (the case of complex conjugates colliding at the origin), and when $n=-m-1$ (collisions on the imaginary axis, away from the origin). There are additional collisions of interest (such as $n=-m-2$, etc.). However, recalling that the perturbations depend periodically on $\mu$ with period 1 , we can omit these additional collisions from consideration.

Thus, we are concerned with locating two different classes of collisions. Following the notation of McLean (1982a) and Francius \& Kharif (2006), Class I collisions 
denote collisions with $n=-m$, while for Class II collisions we have $n=-m-1$. Thus, the interesting eigenvalue collisions are those that satisfy

$$
\begin{aligned}
\text { Class I: } & \lambda_{m}^{-}=\lambda_{-m}^{+}, \\
\text {Class II: } & \lambda_{m}^{-}=\lambda_{-m-1}^{+} .
\end{aligned}
$$

For a fixed value of $m$, we are interested in those values of $\mu$ that correspond to the collision of eigenvalues with opposite signatures by numerically solving these equations for Class I and II instabilities. We use these values of $\mu$ as seeds in $[0,1 / 2]$ around which many values of $\mu$ are chosen, so as to track the location of any possible instabilities. As the amplitude is increased, this location may change, and the set of $\mu$ values is adapted, using what is, in essence, a continuation approach.

\subsection{Water wave spectra}

Using the method outlined in the previous section, we calculate the full spectrum associated with a particular travelling wave with various parameters (depth, amplitude, etc.). To our knowledge, the spectra presented in this section are the first full stability spectra (in a finite region of the complex plane, of course) of periodic travelling wave solutions of the Euler equations presented in the literature.

\subsubsection{Water of infinite depth}

The first spectrum that we examine is the spectrum of a small amplitude wave on water of infinite depth. This spectrum is shown in figure 7(a). A figure-eight curve of eigenvalues associated with instabilities is visible at the origin. We refer to the instabilities associated with the eigenvalues on such figure-eight curves as modulational instabilities. Indeed, at the origin, $\mu=0$. Close to the origin along the figure-eight curve, $\mu$ is small, leading to perturbations with periods close to that of the travelling wave solution. Their superposition leads to modulations of the travelling wave, through the well-known phenomenon of beats. Note that for larger amplitudes, additional regions of non-imaginary eigenvalues are visible. These are commented on below. Overall, it appears that solutions of larger amplitude are susceptible to instabilities with larger growth rates.

As an aside, we also display in figure 7(b) the spectrum for a periodic travelling wave solution of the focusing nonlinear Schrödinger equation (NLS). The NLS equation is an asymptotic equation describing the envelopes of waves in deep water. Like waves in deep water, its solutions are also known to exhibit modulational instabilities, see Zakharov (1968) and Grimshaw (2005). We choose to display the spectrum of the so-called cnoidal wave solution of NLS, since this solution has zero average. We note the qualitative similarity between the two spectra.

To compare our results with those of McLean (1982b), we examine the spectrum for a moderately large amplitude wave $(a k=0.3390)$ associated with two particular Floquet parameters; $\mu=0.0001$ and $\mu=0.4$. For $\mu=0.0001$, we see that there is no unstable eigenvalue confirming that the Benjamin-Feir instability detaches from $\mu=0$ as the amplitude of the travelling wave increases above a critical threshold.

For $\mu=0.4$, we obtain similar maximum growth rates to those presented in McLean $(1982 b)$ for $p=0.6$. Note that under the periodicity constraints, the growth rates associated with $p=0.6$ in the paper by McLean are equivalent to the complex conjugate of the growth rates found for $\mu=0.4$. Furthermore, our method only requires 16 modes for the maximal growth rate to converge to 10 digits of accuracy, whereas McLean (1982b) requires 30 modes to converge to 4 digits of accuracy, see table 2. 



FiguRE 7. (a) Spectrum for the water wave problem with $h=\infty$, for travelling waves of various amplitudes: in order $a=0.01, a=0.095, a=0.183, a=0.223, a=0.262$ and $a=0.299$. (b) Spectrum of a cnoidal wave solution of the NLS equation. 


$\begin{array}{rll}N & \mu=0.0001 & \mu=0.4 \\ 4 & 0.013624 & 0.023333 \\ 8 & 0.002806 & 0.023064 \\ 12 & 0.000626 & 0.023001 \\ 16 & 0.000166 & 0.023000 \\ 20 & 0.000028 & 0.023000 \\ 24 & 0.000000 & 0.023000 \\ 28 & 0.000000 & 0.023000 \\ 32 & 0.000000 & 0.023000\end{array}$

TABLE 2. The real part of the maximum growth rate for $h=\infty$ and $a \approx 0.3390$.
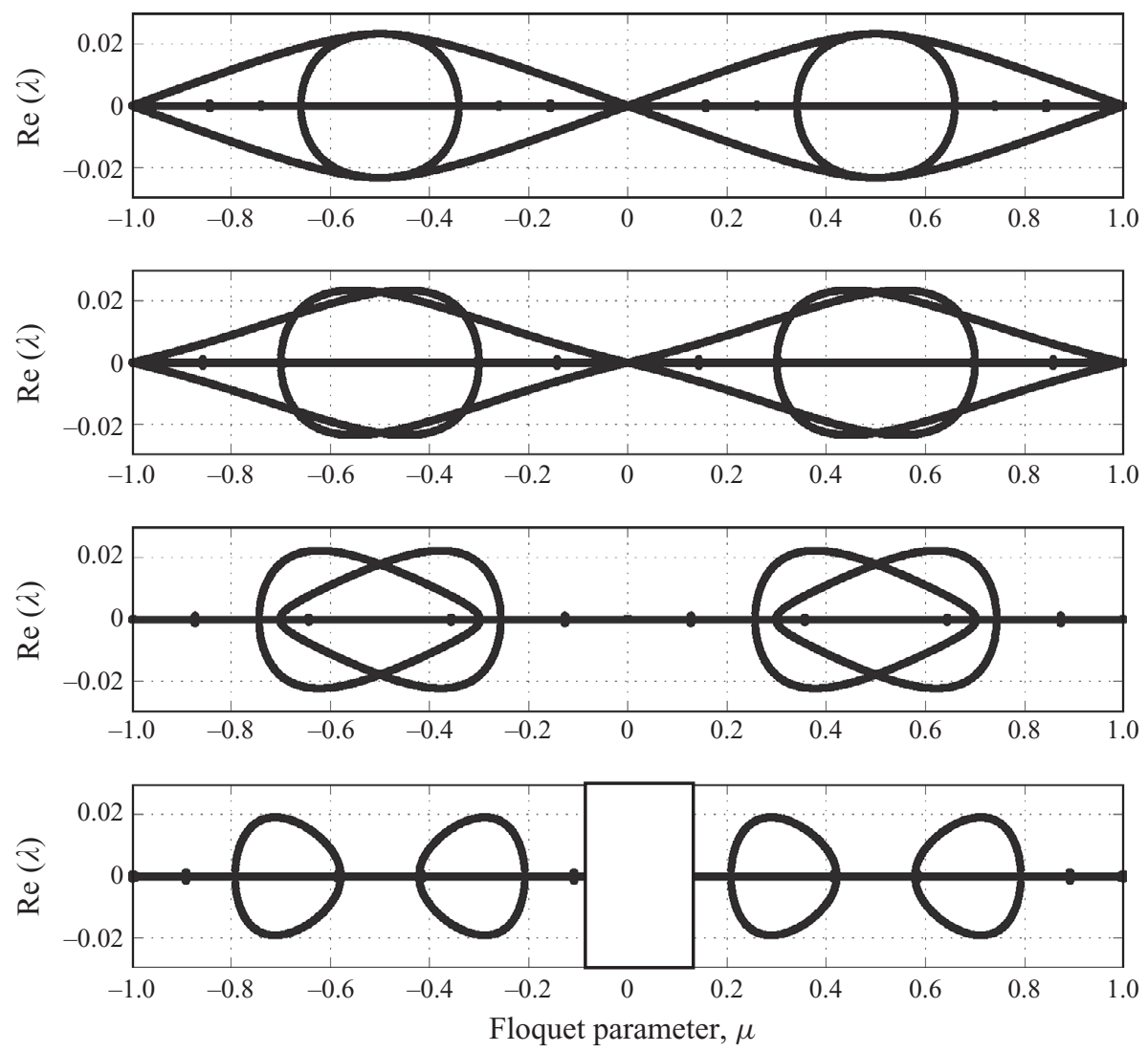

FIGURE 8. Real part of growth rates as a function of the Floquet parameter $\mu$ for $h=\infty$ and increasing amplitudes $a=0.314$ (top), $a=0.334, a=0.354$ and $a=0.374$ (bottom).

As the amplitude of the underlying wave increases, the instabilities continue to separate from $\mu=0$. However, the maximum growth rate decreases exhibiting the same phenomenon found in Longuet-Higgins (1978b) and McLean (1982b). We demonstrate this by plotting the real part of all growth rates $(\operatorname{Re}(\lambda))$ for increasing amplitude waves in water of infinite depth $(h=\infty)$. As shown in figure 8 , as $a k$ increases, the real-valued growth rates separate from $\mu=0$ while they decrease in magnitude. 

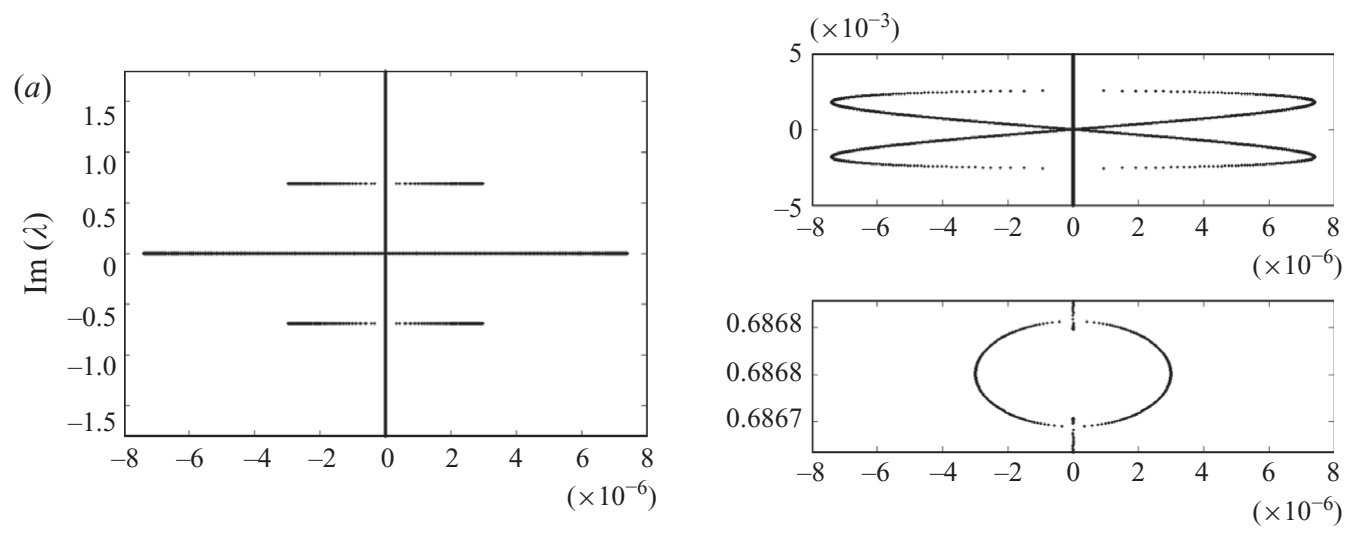

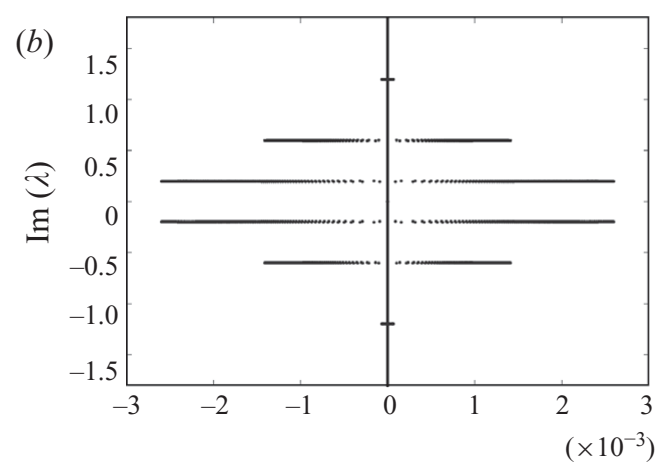

$\operatorname{Re}(\lambda)$
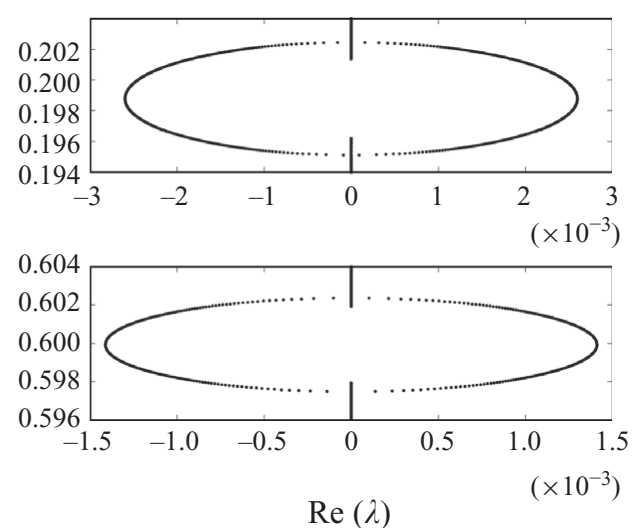

$\operatorname{Re}(\lambda)$

FIGURE 9. ( $a$ ) Spectrum for $h=1.5$ and $a=0.01$ (left). Enlargements are shown (right) for the region near the origin (top) and on the imaginary axis near $0.68 \mathrm{i}$ (bottom). (b) Spectrum for $h=0.5$ and $a=0.01$ (left). Enlargements are shown (right) for the region near 0.1489 i (top) and $0.5212 \mathrm{i}$ (bottom).

\subsubsection{Deep water of finite depth}

To further illustrate the method, we calculate the spectrum associated with a travelling wave solution in water of finite depth $h=1.5$, which is above the Benjamin-Feir threshold. The solution has amplitude $a=0.01$ and it suffices to use 32 Fourier modes. The spectrum and two zooms of it are displayed in figure $9(a)$.

Two main instabilities are visible in figure $9(a)$. The dominant instability (corresponding to the eigenvalue with the largest real part) is associated with a region around the origin. Zooming in on this region shows that it is, once again, a figureeight curve (figure $9 a$, top right), associated with the modulational instability similar to what we observed in water of infinite depth in figure 7 . The other instability region is found near $0.68 \mathrm{i}$ (and near $-0.68 \mathrm{i}$, due to the symmetry of the problem) on the imaginary axis. This region is shown enlarged in figure $9(a)$ (bottom right). It contains an oval of eigenvalues with non-zero real part, leading to instabilities. We refer to this instability (and other similar instabilities associated with such bubbles on the imaginary axis) as 'high-frequency instabilities' since the corresponding perturbations oscillate in time. This is in contrast to the modulational instabilities which correspond to growth rates with small or zero imaginary parts. We discuss the nature of the associated perturbations in more detail below. The only Class I instabilities shown in 


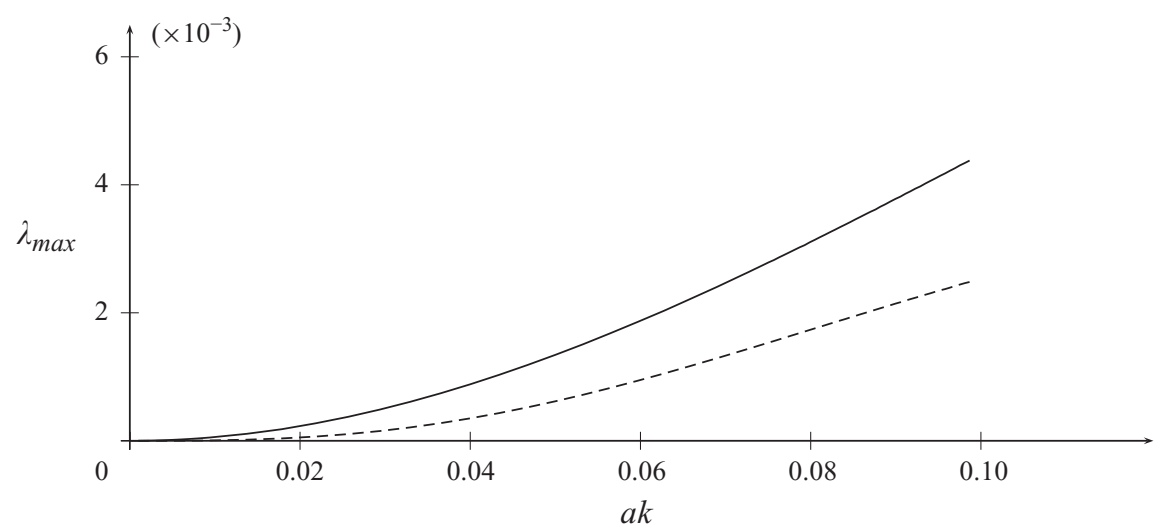

FIGURE 10. The bifurcation curve for the maximum growth rate as a function of amplitude corresponding to Class I (solid line) and Class II (dashed line) for $h=0.5$ and $m=1$.

figure 9 are associated with $m=0$. Numerically, we observe that the magnitude of the Class I instabilities for $h=1.5$ decreases with increasing values of $m$, and thus a faster growing high-frequency instability than the one displayed in figure $9(a)$ is not found.

\subsubsection{Shallow water}

The third spectrum computed corresponds to a travelling wave solution of amplitude $a=0.01$ on water of depth $h=0.5$, which is below the Benjamin-Feir threshold. As before, 32 Fourier modes suffice. The spectrum is shown in figure $9(b)$.

For $h=0.5$ and $a=0.01$, several regions around the imaginary axis display the presence of eigenvalues giving rise to instabilities as seen in figure $9(b)$. Zooming in on the regions of the spectrum associated with these instabilities, we see again that each region contains a smooth curve as seen on the right in figure $9(b)$.

As mentioned above for the trivial solution, there are countably infinite Floquet parameters for which instabilities can occur. For this relatively small amplitude wave (approximately $5 \%$ of the limiting wave height), we can quickly conclude that the travelling wave solution is unstable with respect to the appropriate perturbations.

Consequently, for $h=0.5$, we find that the same instabilities persist for arbitrarily small amplitudes. Figure 10 shows the real part of the Class I and Class II instabilities (where $m=1$ ) as a function of amplitude for $h=0.5$. The corresponding growth rates are order $(a k)^{2}$ and $(a k)^{3}$, respectively. For the Class I instability, this growth rate is equivalent to the corresponding growth rate of the Benjamin-Feir instability as found numerically in McLean et al. (1981).

We only present sample spectra for three different depths as the spectra for other parameters within these categories look similar. Nevertheless, investigating how the spectrum varies as a function of problem parameters is insightful. In the next section, we address the qualitative behaviour of the spectrum as the depth of the fluid is varied.

\subsection{Varying the depth}

A further demonstration of the numerical method is the easy capture of the Benjamin-Feir instability as a function of the dimensionless depth $k h$. Recall that in our numerical experiments $k=1$, since the period $L=2 \pi$. As mentioned in the Introduction, the observation of the Benjamin-Feir instability sparked interest in the stability analysis of Stokes waves. Since the work of Benjamin (1967) and 


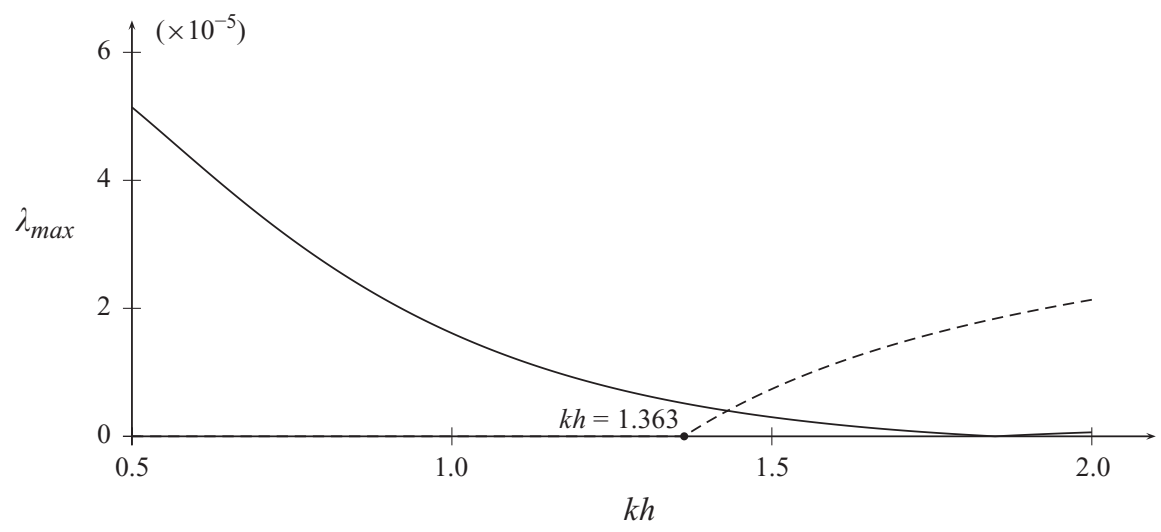

FIGURE 11. Maximal growth rates as a function of dimensionless depth for $a=0.01$ for the high-frequency instability (solid line) compared with the Benjamin-Feir Instability (dashed line).

Whitham (1967), there have been numerous experimental, numerical and analytical investigations to support the instability of Stokes waves when the depth is greater than a critical depth. This instability arises when Stokes wave solutions in dimensionless depth $k h>1.363$ are perturbed with long-wavelength perturbations $(\mu \ll 1)$ as shown by Bridges \& Mielke (1995) for the full set of Euler's equations. For all $k h$ smaller than this critical threshold, Stokes waves are stable to modulational perturbations (i.e. perturbations corresponding to eigenvalues with small $\mu$ values).

Our initial goal when investigating instabilities as a function of $k h$ is to show that the eigenvalues corresponding to long-wavelength perturbations leave the imaginary axis when $k h$ increases past the critical threshold $k h=1.363$. Choosing an arbitrary small wave amplitude $a=0.01$ and a wave period $L=2 \pi$, we computed the corresponding travelling wave solution for a range of depths from $h=0.5$ to $h=2$. Next, we calculated the spectrum for small values of $\mu$ (corresponding to long-wave perturbations) and plotted the real part of the most unstable eigenvalue, as well as the corresponding eigenfunction, In fact, because the system is Hamiltonian, there is a quadrafold symmetry of the spectrum about the origin in the complex plane. Thus, for any eigenvalue $\lambda$, there are three additional eigenvalues $\lambda^{*},-\lambda$ and $-\lambda^{*}$, each with their associated eigenfunctions. When we present an eigenfunction, we take a linear combination of the four eigenfunctions corresponding to the symmetric eigenvalues, to generate a real-valued perturbation. As is seen in figure 11, the most unstable eigenvalue due to long-wave perturbations remains undetectable until $h$ is increased above the critical threshold $h=1.363$ (dashed line).

A surprising result is that once the depth of the fluid is increased beyond the critical depth $h=1.363$, the Benjamin-Feir instability is not immediately dominant. The highfrequency instability growth rate, while decreasing in magnitude as a function of $h$, is larger than the modulational instability growth rate for $h$ close to 1.363. For $h>1.4306$, the modulational instability becomes dominant, as seen in figure 11 . It may appear counterintuitive that the growth rate for the high-frequency instabilities continues to increase as the depth decreases. This is understood by noting that a solution of constant amplitude ( $a=0.01$ for figure 11) becomes relatively more nonlinear as the depth decreases. Lastly, it should be remarked that there exist an infinite number of high-frequency instability growth rate curves in addition to the curve displayed in figure 11, corresponding to the other bubbles emanating higher-up 
(a)

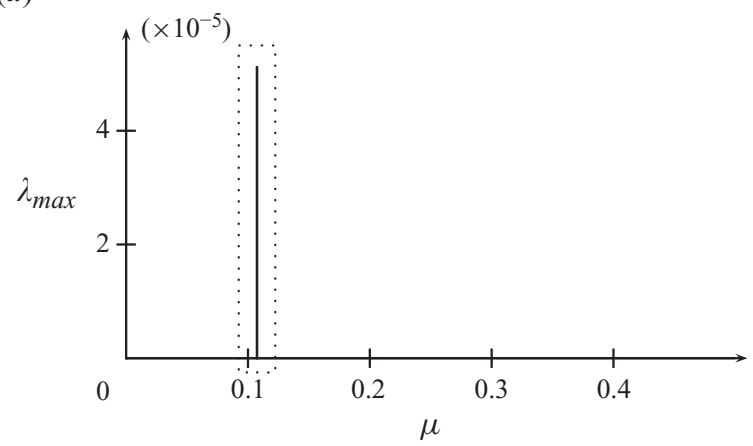

(b)

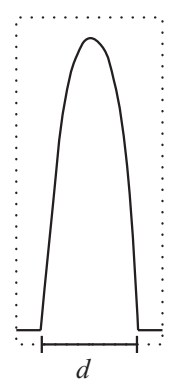

Figure 12. Maximal real part of the spectrum for $h=0.5$ and $a=0.01$ as a function of Floquet exponent $\mu$ associated with the first collision of eigenvalues, i.e. the first bubble. The image in $(b)$ demonstrates that the spike in $(a)$ is the result of a continuous range of $\mu$ values. The width of the curve is $d=0.0002$. Thus, any $\mu$-grid resolution larger than this value could result in a conclusion of stability, whereas the wave is indeed unstable.

on the imaginary axis. These curves have not been included as for most values of the depth their effect is smaller compared to that associated with the first bubble. One effect that is noticeable in figure 11 occurs around $h=1.8$. At this point, the eigenvalue on the first bubble with largest real part is imaginary. In other words, the bubble has collapsed. As the depth increases more, the bubble immediately grows again, and it appears that the eigenvalues with largest and smallest (most negative) real parts merely trade places, leading to smooth curves intersecting on the horizontal axis in figure 11 . At this value of the depth, the growth rates associated with other bubbles dominate that of the first bubble, but they themselves are dominated by the Benjamin-Feir growth rate. For all depths, figure 11 displays at least the dominant growth rate.

\subsection{Discussion}

First, we discuss the nature of the high-frequency instabilities and their associated eigenvalues in more detail. As was pointed out before, computationally these eigenvalues are easily overlooked. As is clear from figure 12, working with a uniformly spaced set of $\mu$ values more than 1/5000 apart would result in missing all unstable eigenvalues. Indeed, the band of unstable eigenvalues has a width of barely $2 \times 10^{-4}$. Other bands tend to have width as small as $10^{-6}$. It is also shown in figure 12 that the high-frequency instabilities are not long-wavelength instabilities. Indeed, the high-frequency instabilities are associated with values of $\mu$ well separated from $\mu=0$.

To the best of our knowledge, there has been no explicit verification of the instability of travelling waves in shallow water in the literature using one-dimensional perturbations of one-dimensional waves. However, other authors have discussed the presence of these high-frequency instabilities in shallow water too, see McLean (1982a), Francius \& Kharif (2006) and Nicholls (2009). The focus of the former two investigations was on the (in)stability of one-dimensional periodic travelling waves with respect to perturbations in the transverse direction, so-called transverse (in)stability. As stated before, our investigations of such perturbations using the nonlocal formulation of Ablowitz et al. (2006) will be reported on in a subsequent paper. While these authors do not explicitly state the presence of instabilities in shallow water with respect to one-dimensional perturbations, they can be inferred from their investigations. They employ a parameter describing the transverse wavelength of the perturbations. Equating this parameter to zero results in one-dimensional 
perturbations, for which it appears their reported instability persists, and agree well with ours. Specifically, we can easily compare the results given in McLean (1982a) and Francius \& Kharif (2006) when the depth is $h=0.5$.

Nicholls (2009) does report on the one-dimensional problem, and investigates for which values of $\mu$ instabilities may arise, using the criterion of MacKay \& Saffman (1986). As his investigations focus on the potential onset of instability, he does not address (i) the magnitude of the instabilities in shallow water, (ii) the range and wavelength of the perturbation and (iii) a comparison of the magnitude of the instability with respect to the well-known Benjamin-Feir instability. All these factors are discussed here.

\subsection{Linear time evolution of the instabilities}

In order to better understand the effect of the high-frequency instabilities, we consider their time evolution using the linear dynamics of the travelling wave solution perturbed by the most unstable eigenfunction. For example, we consider the travelling wave solutions corresponding to $a=0.01$, at the depths $h=0.8$ (shallow water) and $h=1.4306$ (deep water). Recalling that our original perturbed solution is of the form

$$
\eta(x, t)=\eta_{0}(x)+\epsilon \mathrm{e}^{\lambda t} \mathrm{e}^{\mathrm{i} \mu x} \eta_{1}(x),
$$

we explicitly calculate the linear perturbation for multiple times $t$. As discussed before, to preserve the Hamiltonian structure of the dynamics, we incorporate the dynamics of all four eigenfunctions corresponding to the quartet of eigenvalues $\left(\lambda, \lambda^{*},-\lambda,-\lambda^{*}\right)$ in order to preserve the real-Hamiltonian structure of the dynamics. Different time steps are shown in figure $13(a, b)$. Since the eigenvalues are unstable, the amplitude of the solutions grows. Since we are using the linearization, we expect that these approximations are only valid for small times, relative to the size of the growth rate. Indeed, continuing this process for too long simply shows a magnified version of the perturbation. Nevertheless, the initial evolution of the instability should be given accurately by the short-term behaviour of perturbed dynamics under the linear equations.

It remains to determine how the instabilities evolve when they leave the regime of the linear dynamics. It is well known that in deep water the Benjamin-Feir instability leads to exponential growth which eventually tapers off. This process repeats in a sequence of modulation and demodulation cycles also seen in the NLS equation, see Yuen \& Lake (1980). Understanding the nonlinear time evolution of the instabilities in shallow water would give us better insight into the behaviour of general solutions to the shallow water wave problem, but it is outside the scope of the present investigation.

\section{Conclusions and future directions}

We have demonstrated the great practicality of the non-local reformulation of the Euler surface water wave problem due to Ablowitz et al. (2006) to compute onedimensional periodic travelling wave solutions, and to investigate the spectral stability of these solutions. Specifically, we were able to reduce the problem of computing periodic travelling waves to that of solving a single non-local scalar equation for the main variable of physical importance, the surface elevation of the profile. We developed a numerical scheme to determine the surface elevation by incorporating a pseudo-continuation method interpolating smaller amplitude solutions to obtain solutions of larger amplitude. 

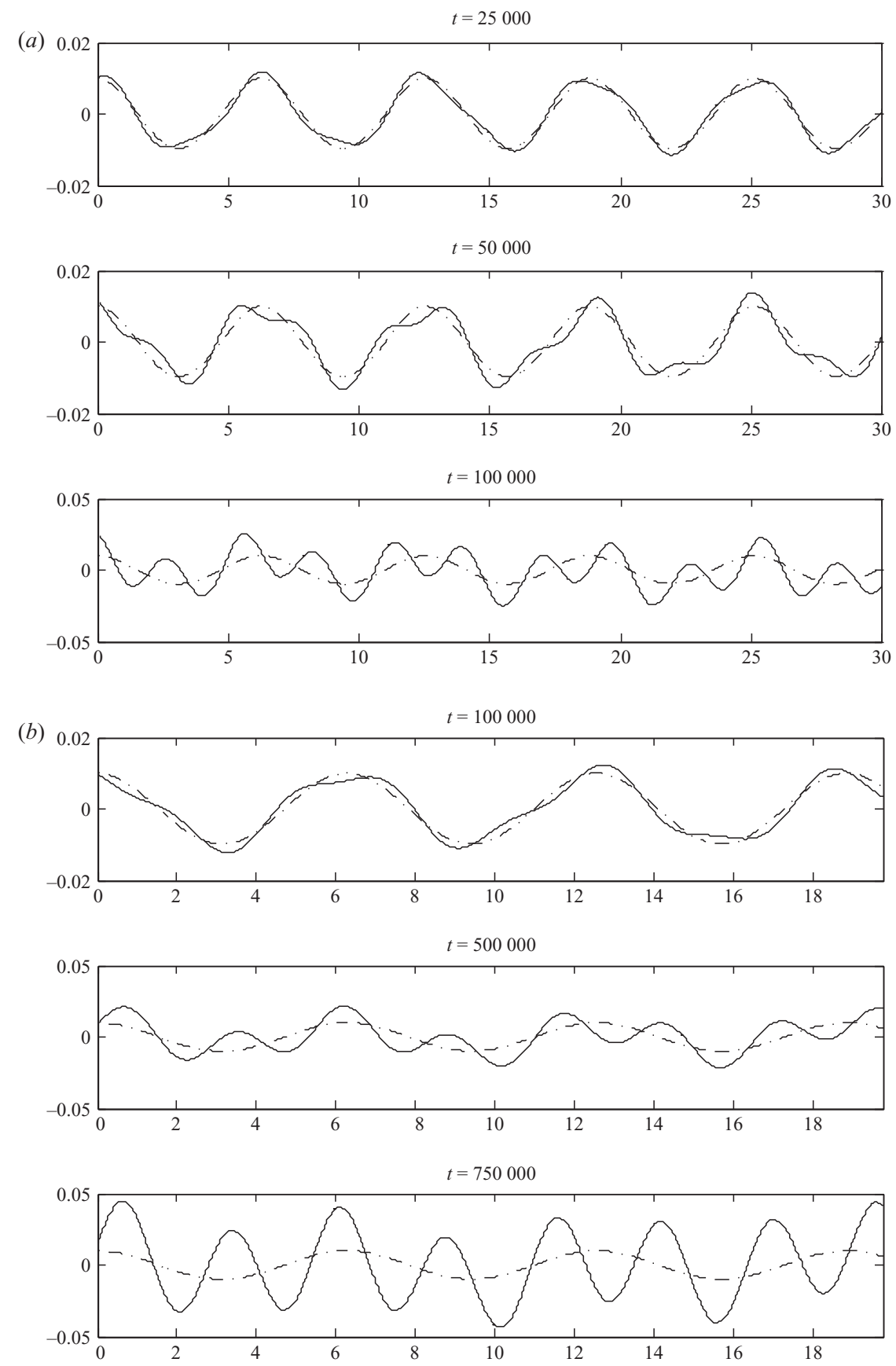

Figure 13. Time evolution of the linear approximation $\eta=\eta_{0}+\epsilon \mathrm{e}^{\mathrm{i} \mu x+\lambda t} \eta_{1}(x)$, where $\eta_{0}$ is the travelling wave solution corresponding to the respective parameter sets. $(a) a=0.01 . h=1.4306$, $\epsilon=0.001, \mu$ is the Floquet exponent corresponding to the most unstable perturbation and $\lambda=3.9916 \times 10^{-6}+0.66843 \mathrm{i}$ and $\eta_{1}(x)$ are the eigenvalue/eigenfunction pair corresponding to the largest growth rate as determined by Hill's method. $(b) a=0.01$ and $h=0.8$, $\epsilon=0.001, \mu$ is the Floquet exponent corresponding to the most unstable perturbation and $\lambda=2.7264 \times 10^{-5}+0.35678 \mathrm{i}$ and $\eta_{1}(x)$ are the eigenvalue/eigenfunction pair corresponding to the largest growth rate as determined by Hill's method. Linear approximations are shown at various time-steps. The dashed line represents the unperturbed wave. 
Using these computed solutions and a Fourier-Floquet decomposition, we analysed the spectral instabilities of the travelling wave solutions for various amplitudes and depths. We chose to investigate the stability of solutions with several depths above and below the critical Benjamin-Feir threshold. As expected, we found that for depths $k h>1.363$ the waves are unstable to long-wavelength perturbations. In addition, we found that the waves are unstable with respect to perturbations with Floquet exponents in specific narrow intervals. In particular, this implies the spectral instability of waves in shallow water. We also found that close to but beyond the Benjamin-Feir threshold, the modulational instability is not dominant. Instead, the high-frequency instabilities that we found are dominant there.

The presence of instabilities in shallow water may be surprising, especially in the face of evidence from various asymptotic models such as the Korteweg-de Vries equation (see Bottman \& Deconinck 2009 and Nivala \& Deconinck 2010) and the Benjamin-Bona-Mahoney equation (see Haragus 2008) among others, demonstrating the stability of periodic waves in the context of these asymptotic models describing waves in shallow water. Those equations are all a consequence of long-wave assumptions, and they are not expected to capture the instabilities represented by the bubble eigenvalues. Experimental evidence as well fails to point towards instabilities in shallow water, see for instance Ablowitz \& Segur (1981) and Wiegel (1982). In addition, the clear presence of the Benjamin-Feir instability in deep water seems to have had a profound effect on the general belief among many in the water wave community that waves in shallow water are stable, since they are not susceptible to this instability.

It remains to put in perspective the effect of the instabilities we found on the dynamics of surface water waves in shallow water. We return to the point that the values of $\mu$ corresponding to the eigenvalues on the bubbles are found in narrow intervals of width on the order of $10^{-6}$, with the largest bubble corresponding to a range of $\mu$ values not exceeding 0.0002 . Recall that, in general, for non-rational values of $\mu$, the perturbations are quasi-periodic, constituting a combination of two signals with non-commensurate wavelengths. If $\mu=p / q$ is rational ( $p, q$ integers), the resulting perturbation is periodic with period $2 \pi q$. In the case of the first bubble, this implies that the minimal period of any unstable perturbation is on the order of $10^{4} \pi$. If one aimed to numerically solve the Euler equations (or one of its alternative formulations) dynamically with the travelling wave and this perturbation as initial condition, a minimal spatial domain of 5000 periods of the travelling wave would be required to examine the dynamics of the perturbation, with good resolution in each of the wavelengths. Given the small size of the growth rate, it is doubtful that any numerical method would be able to achieve enough accuracy to verify the presence of the instability. In other words, the direct numerical verification of the presence of the high-frequency instabilities through the dynamical solution of the Euler equations is essentially unfeasible. A similar statement seems in order for the experimental detection of the high-frequency instabilities. No experimental facility is capable of evolving 5000 wavelengths of a perturbed periodic wave, let alone letting it evolve for a sufficiently long time to observe the small growth rate predicted. For field experiments, the experimental 'facility' has a size on the order of thousands of kilometres, but a shallow water wave in an ocean setting has a wavelength on the order of hundreds of kilometres. It appears that once more the size of the spatial domain is insufficient. On the other hand, in an experimental setting, no restriction is imposed by the boundary conditions, and quasi-periodic perturbations are possible. Since such perturbations simply consist of the simultaneous contribution of two incommensurate 
frequencies, they are easily generated in the lab, and might be observable in the field.

Several answers come to the fore. It is possible that the high-frequency instabilities are a mathematical artifact of considering Euler's equation as our model for surface water waves, without the incorporation of effects such as surface tension and damping. Perhaps these instabilities are not present when these additional effects are considered. Our investigations of capillary-gravity waves using the formulation of Ablowitz et al. (2006) are forthcoming. But it is possible that the high-frequency instabilities are physically relevant. Although the instabilities from periodic perturbations may exist only on scales that are too large for us to observe, either numerically or experimentally, quasi-periodic perturbations might be observable in an experiment.

Many directions are left open for future work. In the immediate future, we will report on our investigations of the transverse instabilities of the one-dimensional solutions. Forthcoming are studies of the stability of capillary-gravity waves, as well as of fully two-dimensional patterns with respect to fully two-dimensional perturbations.

We wish to thank M. Ablowitz, B. Akers, C. Kharif and D. Nicholls for useful conversations. In addition, the referees are thanked for many useful suggestions. This work was supported by the National Science Foundation through grants NSF-DMS0604546 (B.D.) and NSF-DMS-VIGRE-0354131 (K.O.). Any opinions, findings and conclusions or recommendations expressed in this material are those of the authors and do not necessarily reflect the views of the funding sources.

\section{REFERENCES}

Ablowitz, M. \& SEgur, H. 1981 Solitons and the Inverse Scattering Transform. Society for Industrial and Applied Mathematics (SIAM).

Ablowitz, M. J., Fokas, A. S. \& Musslimani, Z. H. 2006 On a new non-local formulation of water waves. J. Fluid Mech. 562, 313-343.

Ablowitz, M. J. \& Haut, T. S. 2008 Spectral formulation of the two fluid Euler equations with a free interface and long wave reduction. Anal. Appl. 6, 323-348.

Benjamin, T. B. 1967 Instability of periodic wave trains in nonlinear dispersive systems. Proc. R. Soc. Lond. A 299, 59-79.

Benjamin, T. B. \& Feir, J. E. 1967 The disintegration of wave trains on deep water. Part 1. Theory. J. Fluid Mech. 27, 417-430.

Benney, D. J. \& Roskes, G. J. 1969 Wave instabilities. Stud. Appl. Maths 48, 377-385.

Bohr, H. 1947 Almost Periodic Functions. Chelsea Publishing Company.

Bottman, N. \& Deconinck, B. $2009 \mathrm{KdV}$ cnoidal waves are linearly stable. DCDS A 25, 1163-1180.

Bridges, T. H. \& Mielke, A. 1995 A proof of the Benjamin-Feir instability. Arch. Rat. Mech. Anal. 133, 145-198.

Chandler, G. A. \& Graham, I. G. 1993 The computation of water waves modelled by Nekrasov's equation. SIAM J. Numer. Anal. 30 (4), 1041-1065.

Cokelet, E. D. 1977 Steep gravity waves in water of arbitrary uniform depth. Phil. Trans. R. Soc. Lond. A 286, 183-230.

Craig, W. \& Nicholls, D. P. 2002 Traveling gravity water waves in two and three dimensions. Eur. J. Mech. B/Fluids 21, 615-641.

Craig, W. \& Sternberg, P. 1988 Symmetry of solitary waves. Commun. Part. Diff. Equ. 13, 603-633.

Craig, W. \& Sulem, C. 1993 Numerical simulation of gravity waves. J. Comput. Phys. 108, 73-83.

Curtis, C. W. \& Deconinck, B. 2010 On the convergence of Hill's method. Maths Comput. 79, 169-187.

Deconinck, B. \& KutZ, J. N. 2006 Computing spectra of linear operators using the FloquetFourier--Hill method. J. Comput. Phys. 219, 296-321. 
Dyachenko, A. I., Kuznetsov, E. A., Spector, M. D. \& Zakharov, V. E. 1996 Analytical description of the free surface dynamics of an ideal fluid (canonical formalism and conformal mapping). Phys. Lett. A 221, 73-79.

Francius, M. \& Kharif, C. 2006 Three-dimensional instabilities of periodic gravity waves in shallow water. J. Fluid Mech. 561, 417-437.

Grimshaw, R. 2005 Nonlinear Waves in Fluids: Recent Advances and Modern Applications. Springer.

Haragus, M. 2008 Stability of periodic waves for the generalized BBM equation. Rev. Roumaine Maths. Pures Appl. 53, 445-463.

Kharif, C. \& Ramamonjiarisoa, A. 1990 On the stability of gravity waves on deep water. J. Fluid Mech. 218, 163-170.

Longuet-Higgins, M. S. 1978 a The instabilities of gravity waves of finite amplitude in deep water. Part I. Superharmonics. Proc. R. Soc. Lond. A 360, 471-488.

Longuet-Higgins, M. S. $1978 b$ The instabilities of gravity waves of finite amplitude in deep water. Part II. Subharmonics. Proc. R. Soc. Lond. A 360, 489-505.

Longuet-Higgins, M. S. 1988 Lagrangian moments and mass transport in Stokes waves. Part 2. Water of finite depth. J. Fluid Mech. 186, 321-336.

MacKay, R. S. \& Saffman, P. G. 1986 Stability of water waves. Proc. R. Soc. Lond. A 406, 115-125.

MCLEAN, J. W. 1982a Instabilities of finite-amplitude gravity waves on water of finite depth. J. Fluid Mech. 114, 331-341.

MCLEan, J. W. $1982 b$ Instabilities of finite-amplitude water waves. J. Fluid Mech. 114, 315-330.

McLean, J. W., Ma, Y. C., Martin, D. U., Saffman, P. G. \& Yuen, H. C. 1981 Three dimensional instability of finite-amplitude water waves. Phys. Rev. Lett. 46, 817-821.

Nicholls, D. P. 1998 Traveling gravity water waves in two and three dimensions. PhD thesis, Brown University.

Nicholls, D. P. 2009 Spectral data for travelling water waves: singularities and stability. J. Fluid Mech. 625, 339-360.

Nivala, M. \& Deconinck, B. 2010 Periodic finite-genus solutions of the KdV equation are orbitally stable. Physica D 239, 1147-1158.

Окамото, H. \& Shолі, M. 2001 The Mathematical Theory of Permanent Progressive Water-Waves. World Scientific Publishing.

Plotnikov, P. I. \& Toland, J. F. 2002 The Fourier coefficients of Stokes' waves. In Nonlinear Problems in Mathematical Physics and Related Topics, I, International Mathematical Series (N.Y.), vol. 1, pp. 303-315. Kluwer/Plenum.

RieneCKer, M. M. \& Fenton, J. D. 1981 A Fourier approximation method for steady water waves. J. Fluid Mech. 104, 119-137.

TANaKa, M. 1983 The stability of steep gravity waves. J. Phys. Soc. Japan 52, 3047-3055.

Vanden-Broeck, J. M. 1983 Some new gravity waves in water of finite depth. Phys. Fluids 26, $2385-2387$.

Whitham, G. B. 1967 Non-linear dispersion of water waves. J. Fluid Mech. 27, 399-412.

Wiegel, R. L. 1982 A presentation of cnoidal wave theory for practical application. J. Fluid Mech. 7, 273-286.

Yuen, H. C. \& Lake, B. M. 1980 Instabilities of waves in deep water. Annu. Rev. Fluid Mech. 12, 303-334.

Zakharov, V. E. 1968 Stability of periodic waves of finite amplitude on the surface of a deep fluid. J. Appl. Mech. Tech. Phys. 9, 190-194. 